\title{
Breast Cancer-Related Neoplastic Alopecia: A Case Report and Review of the Literature
}

\author{
Roberta Vezzoni ${ }^{a}$ Ludovica Toffoli ${ }^{a}$ Claudio Conforti ${ }^{a}$ Arianna Dri ${ }^{a}$ \\ Chiara Retrosi $^{a}$ Nicola di Meo $^{a} \quad$ Giovanni Magaton Rizzi ${ }^{a}$ Diego Signoretto ${ }^{b}$ \\ Iris Zalaudek ${ }^{a}$ \\ aDermatology and Venereology Department, Maggiore Hospital of Trieste, University of Trieste, Trieste, Italy; \\ ${ }^{b}$ Department of Histopathology, Cattinara Hospital of Trieste, Trieste, Italy
}

\section{Keywords}

Alopecia · Dermatopathology · Dermoscopy · Skin cancer

\section{Abstract}

Neoplastic alopecia (NA) is defined as an organized hair loss in single or multiple areas of the scalp caused by a primary tumor that has metastasized to the skin of the scalp. Due to its localization and clinical appearance, NA should be placed in differential diagnosis with alopecia areata or other entities. To date, pathognomonic dermoscopic criteria of NA have not yet been described: the absence of classical criteria of other scalp diseases in addition to a major neovascularization with on-focus arborizing vessels and erosions or ulcerations may help the clinician to suspect a diagnosis of secondary alopecia. Dermatologists should pay more attention to these rare forms of secondarism because in exceptional cases, a simple alopecia of the scalp can hide a new, relapsing or metastatic neoplasia.

๑) 2021 S. Karger AG, Basel

\section{Introduction}

Neoplastic alopecia (NA) is defined as an organized hair loss in single or multiple areas caused by a primary tumor that has metastasized to the skin of the scalp. In $84 \%$ of cases, NA is linked to primary breast cancer [1]. The mechanism underlying skin metastatization is not yet known, and it seems to have a multifactorial origin. Clinical presentation of NA can be various, as its range of differential diagnosis encompasses many causes of alopecia. Among these, alopecia areata (AA), frontal fibrosing alopecia (FFA), discoid lupus erythematosus (DLE), and lichen planopilaris (LPP) are the most relevant. Due to its localization and clinical appearance, in our case, NA should be placed in differential diagnosis with AA [1], and this is the reason why dermoscopy has been an extremely useful tool for the correct differential diagnosis among these entities [2,3].

In this review, we present the results obtained from the analysis of 27 cases of NA associated with breast cancer, in order to focus on the prevailing modalities of manifestation of NA. All the patients were woman.

Correspondence to:

Arianna Dri, arianna.dri@gmail.com (c) 2021 S. Karger AG, Basel

$$
\text { v'⿳亠丷厂 }
$$




\section{Case Report}

An 85-year-old otherwise healthy woman was referred to our skin cancer department for the evaluation of a progressive and recently onset alopecia. The dermatological examination showed a localized area of complete hair loss of the parieto-occipital region of the scalp (shown in Fig. 1a) of about $10 \times 8 \mathrm{~mm}$ diameter, with fibrotic consistency and well-defined borders and an evident neovascularization with fine telangiectasias and crusted lesion. The dermoscopic evaluation did not show classical criteria of AA (i.e., yellow dots, black dots, broken hairs, short vellus hair, and tapering hairs), but it showed giant on-focused arborizing vessels and fine telangiectasias on a pink-whitish background and a single well-defined orangish lesion with polymorphic vessels surrounded by a yellow-white crust (shown in Fig. 1b). Due to the nonunivocity of clinical and dermoscopical presentation, a punch biopsy was performed. The histopathological report showed a neoplasm compatible with a secondary localization of ductal breast cancer (shown in Fig. 2).

The clinical history of the patient revealed a previous left breast infiltrating ductal carcinoma treated with quadrantectomy, axillary lymphadenectomy, and radiotherapy in 2002, and the last oncologic follow-up in 2006 was negative for recurrences. The patient underwent mammography and ultrasound examination of breasts, which revealed the presence of 2 lesions of $15 \mathrm{~mm}$ in the super-external quadrant and $8.5 \mathrm{~mm}$ in the external paraareolar quadrant compatible with metastasis (shown in Fig. 3). A first-line hormone treatment with exemestane $25 \mathrm{mg} 1 \mathrm{cp} /$ day was started.

\section{Clinical Features of NA}

Several clinical variants of NA have been described in the literature. It mostly appears as single or multiple reddish painless patches or plaques, variable in size, up to a maximum extension of $80 \mathrm{~cm}^{2}$, even though universal alopecia has also been reported [1, 4]. In smaller percentages, NA presents as flesh-colored nodules. Ulceration was found in 5 cases, and occasionally lesions were accompanied by symptoms such as itching and stinging. In nearly half of the cases (41\%), erythema is observed, a sign that could distinguish a pattern of secondary alopecia from an AA [4].

A prevailing location has not been identified, and in fact, areas of NA manifested all over the scalp, having been reported in the frontal, parietal, occipital, and vertex region. In 2 noteworthy cases, alopecia occurred accompanied by other peculiar skin lesions attributable to primary neoplasm: a patient presented telangiectatic lesions on the forehead, nose, and cheeks, while another patient presented a zosteriform eruption of the trunk, both in concomitant association with areas of alopecia $[5,6]$ (shown in Table 1).

\section{Scalp Dermoscopy}

Dermoscopy can be a valid and easy technique for diagnosis and management of alopecia. To date, there are rare cases of NA in the literature, and the dermoscopic findings are infrequently described; moreover, the lesion can mimic other diseases or exhibit doubtful features.

AA shows usually yellow dots, dystrophic hairs, and clustered short vellus hairs. Yellow dots are the most sensitive markers for the diagnosis, but they are not very specific, as they can be seen in other diseases. Black dots (cadaverized hairs), broken hairs, and

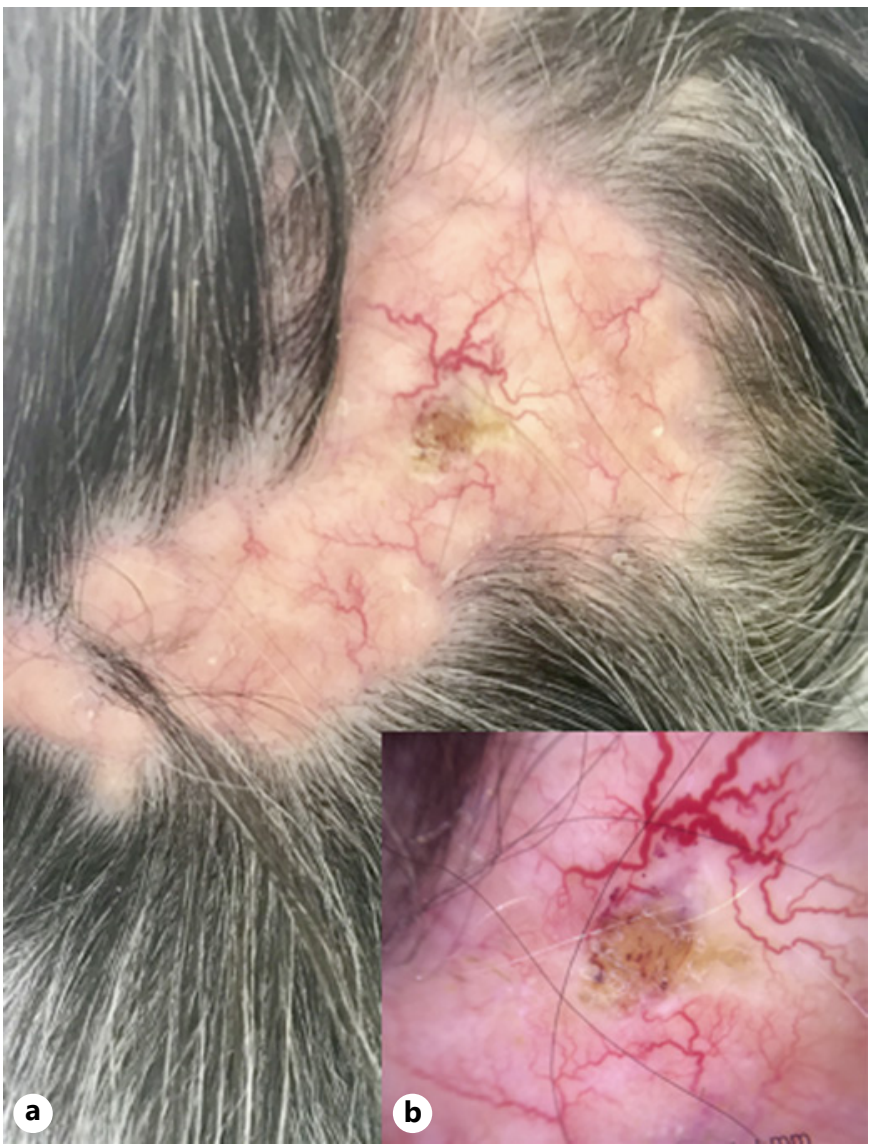

Fig. 1. a Clinical appearance of NA due to breast cancer with a 10 $\times 8$-mm plaque of the parieto-occipital region with neovascularization. b Dermoscopy features of NA showing giant on-focus arborizing vessels in a pink-whitish background with an orangish well-defined lesion of about $5 \mathrm{~mm}$ in diameter. NA, neoplastic alopecia.

tapering hairs are usually present and are associated with disease activity, and exclamation-mark hairs are the most specific signs of acute AA, even if they can be present in chemotherapy alopecia. Other hair shaft abnormalities present in AA are coudability hairs, pseudo-monilethrix, and coiled hairs $[27,28]$.

In the androgenetic alopecia, dermoscopy shows vellus hair, peripilar signs, and hair diameter variability. Advanced androgenetic alopecia most commonly presents yellow dots, empty follicular ostia, and, in bald sun-exposed areas, honeycomb-pigmented network [28].

The characteristics of LPP are the absence of follicular openings and the presence of whitish perifollicular scales; the follicle fibrosis is associated with the presence of white dots [27].

An uncommon type of LPP is FFA that shows a progressive recession of the frontotemporal hairline, with atrophic and smooth skin and absent follicular openings. Around the terminal hairs, erythema and follicular hyperkeratosis may be present, and the absence of vellus hair is very diagnostic of FFA. Other typical findings are the lonely hair sign, pili torti, black dots, broken hairs, and eyebrows loss [29]. 
Fig. 2. a Single file carcinomatosis elements, $\times 20$. b Evidence of pagetisation phenomena, the expression of CK7 is oriented towards the epithelial origin of the neoplasm, $\times 20$.

Fig. 3. a Breast ultrasound showing a 15$\mathrm{mm}$ hypoechogenic solid lesion of the super-external quadrant compatible with the secondary lesion of previous breast cancer. b Diagnostic mammography of the right breast. A well-defined contrast area, with a diameter of about $15 \mathrm{~mm}$ of the super-exterior quadrant and in the external paraareolar, $8.5 \mathrm{~mm}$.
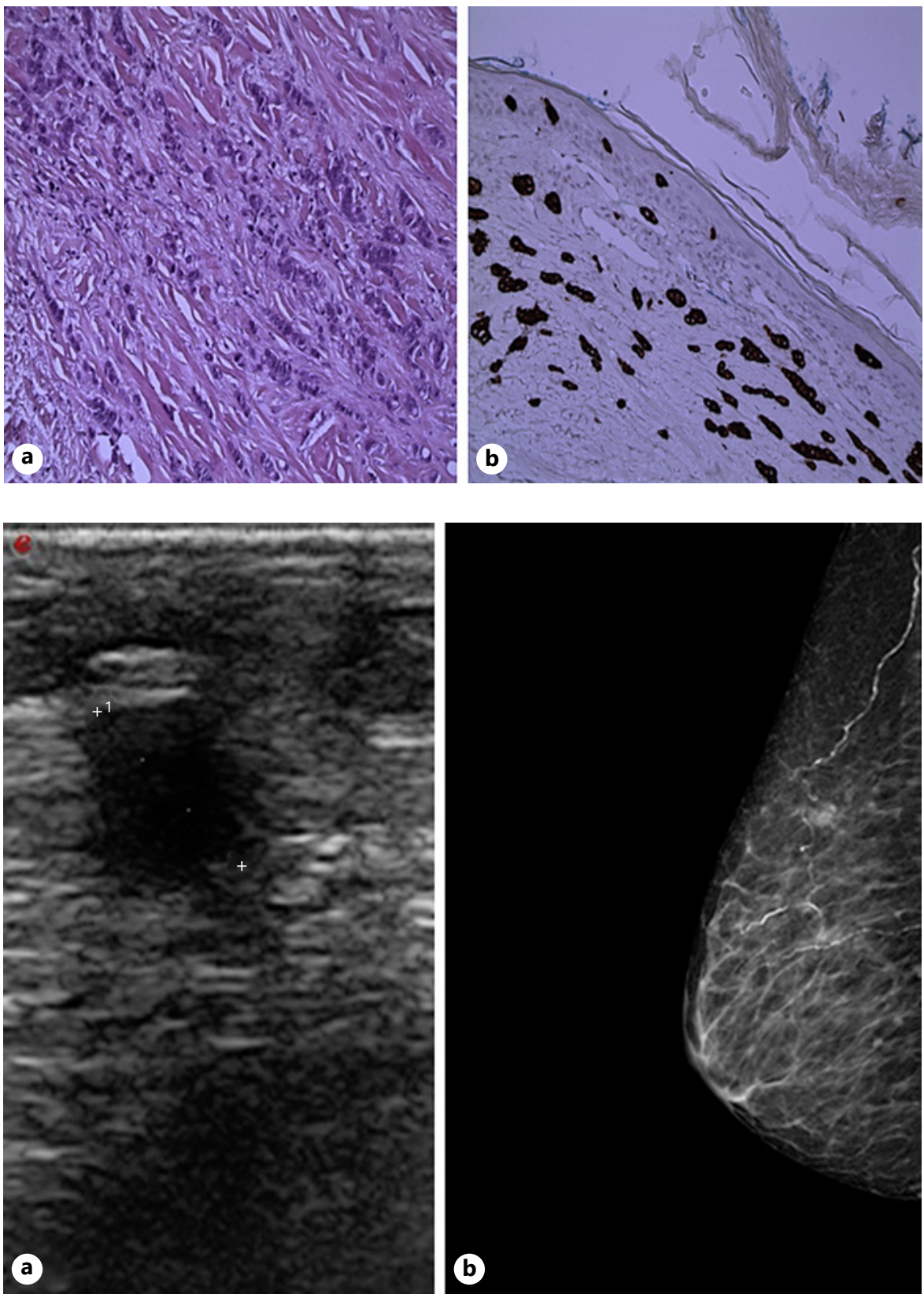

Specific findings of DLE of the scalp are keratotic plugs, red dots, and enlarged branching vessels. Peripilar concentric white scales and peripilar erythema may be present. The absence of follicular openings and the cicatricial white patches are observed both in DLE and LPP [29].

In our case, dermoscopy reveals a dense network of neovascularization with giant on-focused arborizing vessels and fine telangiectasias on a pink-whitish background and a single well-defined orangish lesion with polymorphic vessels surrounded by a yellowwhite crust. The suspect of NA may arise from the absence of classical dermoscopic criteria of hair disorders and the presence of features associated with malignancy.

The Role of Dermoscopy in the Diagnosis of Neoplastic Alopecia

\section{Latency}

NA can present as the first sign of an undiagnosed neoplasia or can occur several years after the diagnosis of the primary tumor in the absence of other symptoms. In particular, NA was the initial presentation of an unknown primary tumor in a limited number of cases, while in most of them appeared after neoplasm onset, over a period of time ranging from 1 to 15 years. Very often the primitive had already been treated, so NA configured in the setting of a relapsing disease. Primitive simultaneous metastases were detected in about $33 \%$ of the cases, identifying a long ago disseminated disease. Dissemination presented simultaneously with NA in nearly $30 \%$ of the patients, among which subcutaneous metastases had relevant frequency (shown in Table 1). 


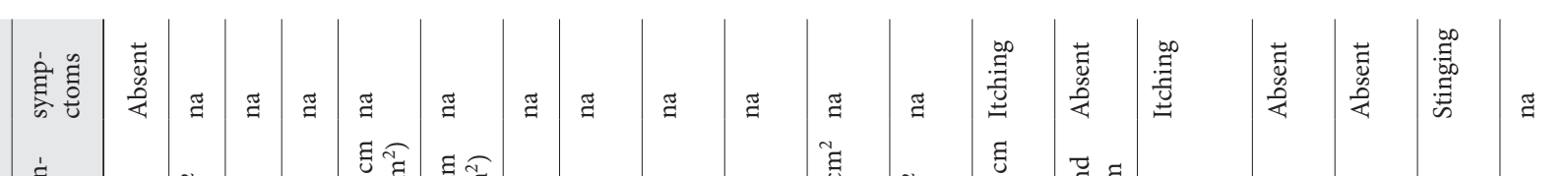

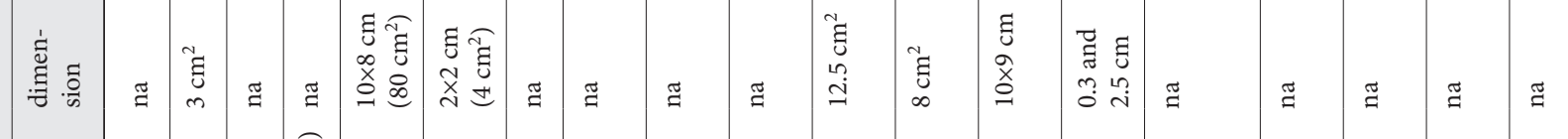

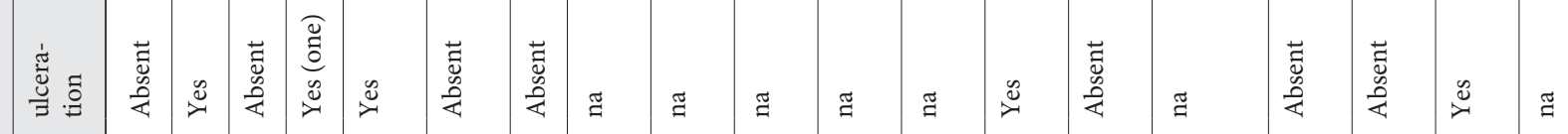

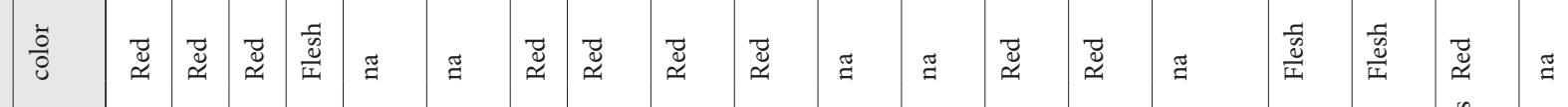

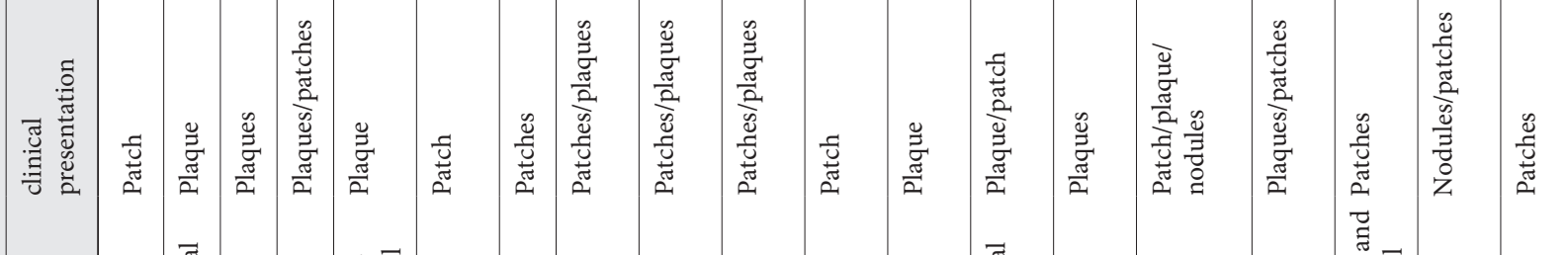

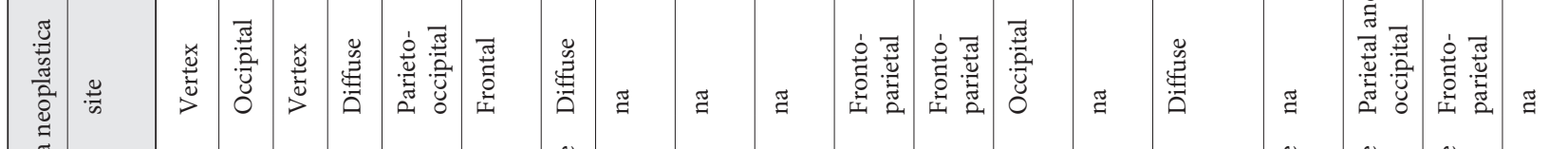

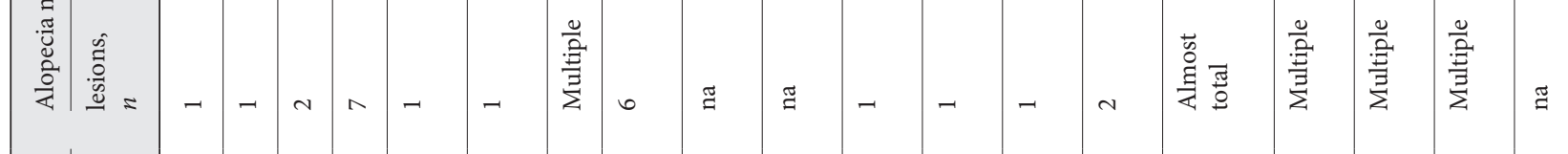

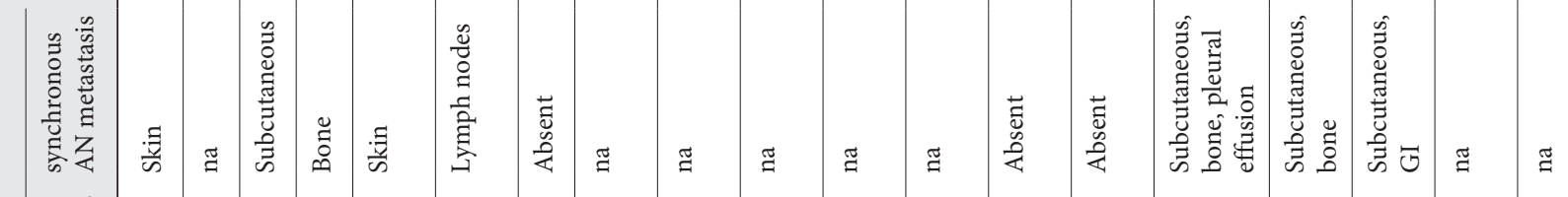

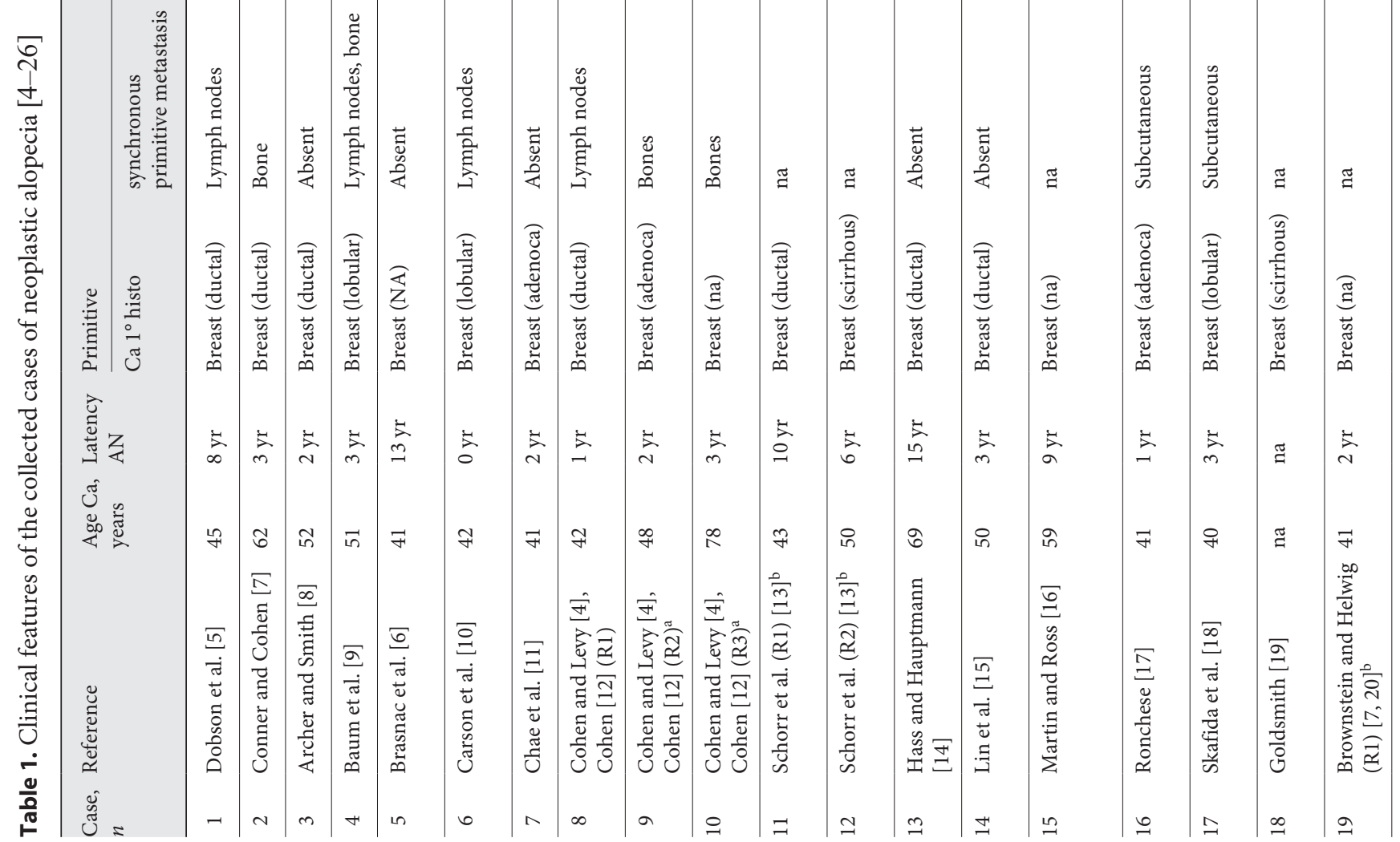




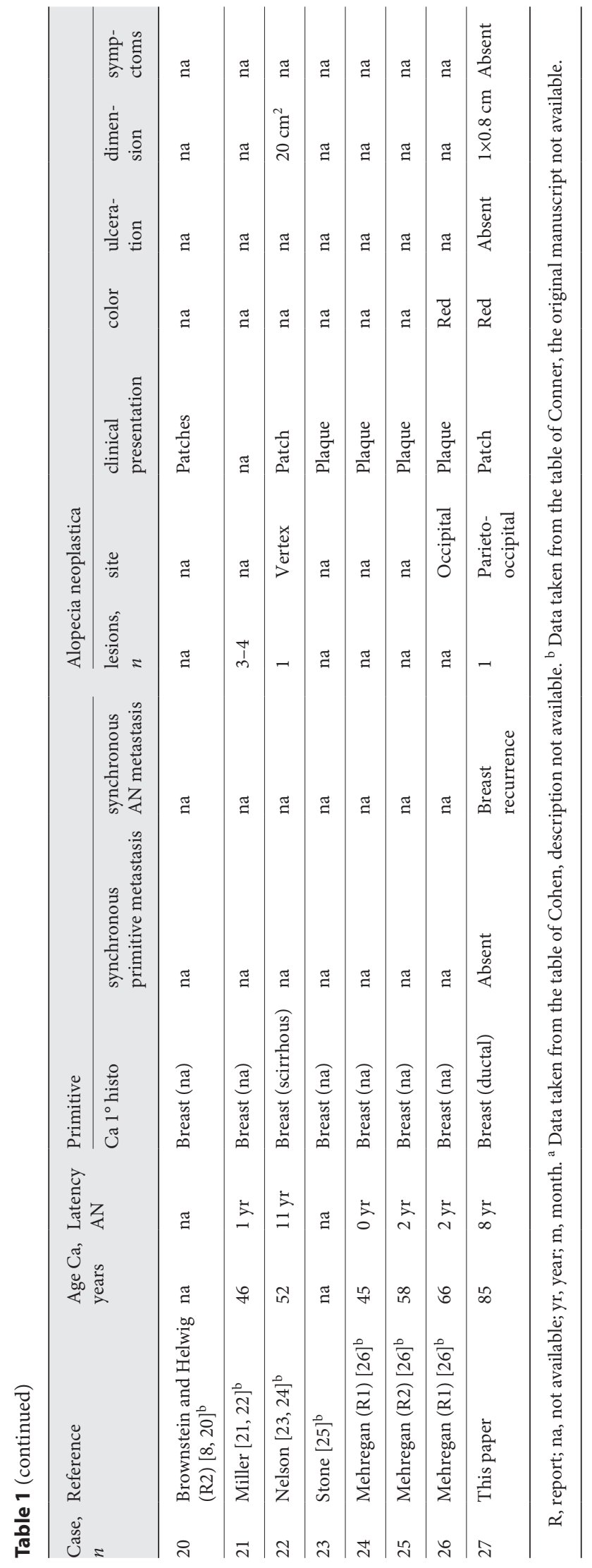

The Role of Dermoscopy in the Diagnosis of Neoplastic Alopecia

\section{Primitive Neoplasia}

Histological subtype of the primitive was ductal infiltrating adenocarcinoma in most cases, observing lobular carcinoma in a smaller number and sporadically scirrhous. It must be said that ductal adenocarcinoma is the most frequent histotype of breast cancer, so it is not surprising that NA occurred more frequently in this setting. This fact makes it difficult to establish clear associations between the type of clinical presentation of NA and primitive histologic subtype. Moreover, the number of cases is still limited (shown in Table 1).

\section{Pathogenesis}

It is estimated that scalp is the site involved by $4-7 \%$ of cutaneous metastasis from visceral malignancies. Tumors can spread to the scalp in various modalities. Regarding breast cancer, these metastases could develop as a result of a retrograde lymphatic dissemination from cervical and supraclavicular nodes, through lymphatic emboli or via hematogenous dissemination. The latter is undoubtedly the most frequent path, in consideration that scalp is provided with a rich vascular network. Pathogenetic mechanism of NA has not yet been fully understood. It usually configures as a scarring process, so the most accredited theory is that pilosebaceous units are destroyed by the desmoplastic reaction due to tumor cells. Some authors postulate that particular fibrogenic cytokines released by neoplastic cells, such as basic fibroblast growth factor, transforming growth factor beta, and interleukins 4 and 6, may contribute to induce atrophy of the follicles, in a way similar to inflammatory alopecia. Anyway, further studies are needed. Compression created in the dermis by tumoral mass itself could compromise survival and development of pilosebaceous units. This would explain the fact that sometimes hair regrew after proper cancer treatment [12, 30-32].

Attempts have been made to understand if there is a correlation between metastatization to the scalp and the histological type of primitive neoplasia. Few data available seem to highlight that adenocarcinoma is the subtype that most frequently associates with AN, perhaps because of the high density of glands in the scalp [32]. However, adenocarcinoma is the most frequent histologic subtype of breast cancer too.

\section{Histopathological Examination}

Histological examination of NA lesions frequently revealed infiltration of tumor cells consistent with primary malignancy embedded in a dense collagenous dermal stroma that can extend to subcutaneous tissue. Pilosebaceous units are visibly atrophic or completely absent [7].

\section{Treatment and Prognosis}

The presence of metastases from visceral neoplasms is often associated with a poor prognosis. However, scalp metastases with associated alopecia have a relatively better outcome with survival measured in years [7]. There is not a standard treatment for NA, and in fact, the therapeutic choice is based on the receptors expressed by the neoplasm, overall staging, and comorbidities of the patient [7]. Main approaches adopted were radiotherapy, chemotherapy, and hormonal therapy, aiming to target both tumor and metastasis (shown in Table 1).

Our patient developed NA 8 years after diagnosis of breastinfiltrating ductal carcinoma as a localized area of complete hair loss of the parieto-occipital region of the scalp. Nodules compati- 
ble with neoplastic recurrences have also been detected on mammography. The patient is currently alive, and she is being treated with exemestane. Therefore, these data are in line with those of the majority of the cases reviewed.

\section{Conclusions}

Clinicians must consider NA in the spectrum of possible differential diagnoses when they have to face with forms of alopecia in which characteristics are not easily classifiable within the classic forms. Atypical anamnestic and clinical features, along with nonresponse to standardized therapies, should raise the index of suspicion. Dermoscopy configures as a very important tool to preliminarily rule out other scalp disorders marked by more specific criteria.

To date, pathognomonic dermoscopic criteria of NA have not yet been described: the absence of classical criteria of other scalp diseases in addition to a major neovascularization with on-focus arborizing vessels and erosions or ulcerations may help the clinician to suspect a diagnosis of secondary alopecia. Dermatologists should pay more attention to these rare forms of secondarism because in exceptional cases, a simple alopecia of the scalp can hide a new, relapsing or metastatic neoplasia. When these situations are suspected, a prompt biopsy is required, as histological examination will provide the definitive diagnosis.

\section{Statement of Ethics}

This manuscript has not been published or submitted for publication elsewhere. The authors state that they have obtained written informed consent to publish the reported case and clinical images.

\section{Conflict of Interest Statement}

The authors declare to have no conflicts of interest for the publication of this article.

\section{Funding Sources}

The authors did not receive any funding.

\section{Author Contributions}

All authors have contributed significantly and seen and approved the final version of the submitted manuscript. Iris Zalaudek, Nicola di Meo, and Giovanni Magaton-Rizzi defined the design and intellectual content of the manuscript. Diego Signoretto provided the histopathological images. Roberta Vezzoni, Ludovica Toffoli, and Arianna Dri wrote the draft, edited the manuscript, figures, and table, and prepared them for submission. Claudio Conforti and Chiara Retrosi reviewed the article.

\section{References}

1 De Giorgi V, Grazzini M, Alfaioli B, Savarese I, Corciova SA, Guerriero G, et al. Cutaneous manifestations of breast carcinoma. Dermatol Ther. 2010 Nov-Dec;23(6):581-9.

2 Conforti C, Giuffrida R, Vezzoni R, Resende FSS, di Meo N, Zalaudek I. Dermoscopy and the experienced clinicians. Int J Dermatol. 2019 Jun 20.

3 Conforti C, Giuffrida R, Retrosi C, di Meo N, Zalaudek I. Two controversies confronting dermoscopy or dermatoscopy: nomenclature and results. Clin Dermatol. 2019 Sep-Oct; 37(5):597-9.

4 Cohen I, Levy E, Schreiber H. Alopecia neoplastica due to breast carcinoma. Arch Dermatol. 1961;84(3):490-2.

5 Dobson CM, Tagor V, Myint AS, Memon A. Telangiectatic metastatic breast carcinoma in face and scalp mimicking cutaneous angiosarcoma. J Am Acad Dermatol. 2003 Apr; 48(4):635-6.
6 Brasanac D, Boricic I, Todorovic V. Epidermotropic metastases from breast carcinoma showing different clinical and histopathological features on the trunk and on the scalp in a single patient. J Cutan Pathol. 2003 Nov; 30(10):641-6

7 Conner KB, Cohen PR. Cutaneous metastasis of breast carcinoma presenting as alopecia neoplastica. South Med J. 2009 Apr;102(4): 385-9.

8 Archer CB, Smith NP. Alopecia neoplastica responsive to tamoxifen. J R Soc Med. 1990 Oct;83(10):647-8.

9 Baum EM, Omura EF, Payne RR, Little WP. Alopecia neoplastica: a rare form of cutaneous metastasis. J Am Acad Dermatol. 1981 Jun;4(6):688-94.

10 Carson HJ, Pellettiere EV, Lack E. Alopecia neoplastica simulating alopecia areata and antedating the detection of primary breast carcinoma. J Cutan Pathol. 1994 Feb;21(1): 67-70.
11 Chae YS, Suh KS, Kim ST. Alopecia neoplastica due to breast cancer. Ann Dermatol. 1990 Jul;2(2):89-92.

12 Cohen PR. Primary alopecia neoplastica versus secondary alopecia neoplastica: a new classification for neoplasm-associated scalp hair loss. J Cutan Pathol. 2009 Aug;36(8): 917-8.

13 Schorr WF, Swanson PM, Gomez F, Reyes $\mathrm{CN}$. Alopecia neoplastica. Hair loss resembling alopecia areata caused by metastatic breast cancer. JAMA. 1970;213(8):1335-7.

14 Haas N, Hauptmann S. Alopecia neoplastica due to metastatic breast carcinoma vs. extramammary Paget's disease: mimicry in epidermotropic carcinoma. J Eur Acad Dermatol Venereol. 2004 Nov;18(6):708-10.

15 Lin WL, Lin WC, Jung SM, Yang CH, Hong HS. Breast cancer metastasized to the scalp mimicking alopecia areata: alopecia neoplastica. Breast J. 2007 Jan-Feb;13(1):94-5. 
16 Martin J, Ross JB. Alopecia totalis as a presentation of cutaneous metastasis (alopecia neoplastica). Int J Dermatol. 1983 Oct;22(8):4879.

17 Ronchese F. Alopecia due to metastases from adenocarcinoma of the breast; report of a case. Arch Derm Syphilol. 1949 Mar;59(3): 329-32.

18 Skafida E, Triantafyllopoulou I, Flessas I, Liontos M, Koutsoukos K, Zagouri F, et al. Secondary alopecia neoplastica mimicking alopecia areata following breast cancer. Case Rep Oncol. 2020 Jun 11;13(2):627-32.

19 Goldsmith WN. A case of metastatic scirrhous carcinoma of the scalp. Br J Dermatol. 1929;41(7):270-3.

20 Brownstein MH, Helwig EB. Metastatic tumors of the skin. Cancer. 1972 May;29(5): 1298-307.
21 Miller JL. Alopecia caused by metastatic carcinoma, presumably from breast. Discussion. Arch Dermatol. 1964;90:249.

22 Miller JL. Alopecia neoplastica (possibly of 28 years' duration). Discussion. Arch Dermatol. 1972;105:120.

23 Nelson CT. Alopecia caused by metastatic carcinoma, presumably from breast. Arch Dermatol. 1964;90:249-50.

24 Nelson CT. Alopecia neoplastica (possibly of 28 years' duration). Arch Dermatol. 1972; 105:120.

25 Stone SP. Cutaneous clues to cancer. Am Fam Physician. 1975;12:82-88.

26 Mehregan AH. Metastatic carcinoma to the skin. Dermatologica. 1961;123:311-25.

27 Rigopoulos D, Stamatios G, Ioannides D. Primary scarring alopecias. Curr Probl Dermatol. 2015;47:76-86.
28 Miteva M, Tosti A. Hair and scalp dermatoscopy. J Am Acad Dermatol. 2012 Nov;67(5): $1040-8$.

29 Iorizzo M, Tosti A. Frontal fibrosing alopecia: an update on pathogenesis, diagnosis, and treatment. Am J Clin Dermatol. 2019 Jun; 20(3):379-90.

30 Scheinfeld N. Review of scalp alopecia due to a clinically unapparent or minimally apparent neoplasm (SACUMAN). Acta Derm Venereol. 2006;86(5):387-92.

31 Crotty K, McCarthy W, Quinn M, McCarthy S. Alopecia neoplastica caused by desmoplastic melanoma. Australas J Dermatol. 2003 Nov;44(4):295-8.

32 Paolino G, Pampena R, Grassi S, Mercuri SR, Cardone M, Corsetti P, et al. Alopecia neoplastica as a sign of visceral malignancies: a systematic review. J Eur Acad Dermatol Venereol. 2019 Jun;33(6):1020-8. 